\title{
The passage of time
}

Eric Olson (2009) has argued that the dynamic view of time must be false. For it requires that the question 'How fast does time pass?' has an answer. But the only possible answer is: one second per second. However, this is not an answer, since 'one second per second' does not denote a rate of change since it does not denote a rate at all. It denotes a number, the number one, since what it denotes is one second divided by one second ('per' means 'divided by one ...'), which is one since dividing anything by itself, unless it is zero, gives you one (Olson 2009:5). But no number is a rate, a fortiori, not a rate of change. So the question 'How fast does time pass?' has no answer. So the dynamic view of time must be false.

This argument is in fact a frequent flier, Olson and Phillips (2010) note earlier occurrences in the literature. But Olson's aim is to put a precise finger on what is wrong with talk of time's passage. What is wrong, he argues, is that there can be no such change as time's passage because there is no such rate.

I first argue that Olson has failed to identify what is wrong with talk of time's passage. Then I argue that, nonetheless, he is right to reject it. To say that time passes is analogous to saying that space is dense, and to ask about the rate of time's passage is analogous to asking how dense space is. ${ }^{1}$ No appeal to the idea that 'per' means 'divided by one ...' is needed to explain the meaninglessness of the question 'how dense is space?' (and its only possible answer: one cubic centimetre per cubic centimetre); hence there is no need to appeal to this idea to explain the meaninglessness of the question 'how fast does time

\footnotetext{
${ }^{1}$ Of course, it is true that I had breakfast five hours ago, an hour ago had breakfast four hours earlier and an hour from now will have had breakfast six hours earlier, and we can choose to restate this fact by saying that the event of my breakfasting was only four hours in the past an hour ago, has passed a further hour into the past in the last hour and will pass a further hour into the past in the next hour. So it is true, in a sense, that time passes. But this fact, however, expressed, must be accepted by both A-theorists and B-theorists. This is not what Olson is rejecting. What he is rejecting, correctly I argue, under the name 'the passage of time', is something which it is supposed that A-theorists can acknowledge but B-theorists cannot, but this is just an illusion of meaningful language, analogous to talk of the density of space.
} 
pass?' (and its only possible answer: one second per second). Nevertheless, since the questions are on a par the dynamic view of time, which requires that they are not, is mistaken.

Iain Phillips (2010) has already replied to Olson by suggesting that he has confused ratios, which are relations between quantities, with quotients, which are numbers. He gives the example of exchanging blue tiles at the rate of one to one. This is, of course, not a rate of change, but it is still a rate, a rate of exchange. By Olson's argument it is not, since it is a number. But it may be denied that in this example 'one blue tile per blue tile' is a rate at all.

However, there are more familiar examples of what are in fact rates that Olson's argument would rule out.

Further north the bison are more massive. The rate of increase is 1 kilogram every kilometre: $1 \mathrm{~kg}$ per $\mathrm{km}$. The wolves are also bigger. The rate of increase is $1 \mathrm{~cm}$ in shoulder height every kilometre: $1 \mathrm{~cm}$ per $\mathrm{km}$, or $1 \mathrm{~cm}$ per $100,000 \mathrm{~cm}$. By Olson's reasoning this is one centimetre divided by 100,000 centimetres, which is just 1/100,000. But this is just a number, not a rate.

Another familiar example to the same purpose: the gradient of a hill, so many feet rise to so many feet run, 1:1 if the gradient is 45 degrees.

Quick Draw McGraw used to be faster on the draw than he is now. Five years ago his draw was clocked as one second (dead). This year it was clocked as 1.5 seconds - a rate of change of 0.1 seconds per year over five years. But this, Olson will say, is just 0.1 of a second divided by one year, which is just 0.1 seconds divided by $(365 \times 24 \times 60 \times 60)$ seconds, which is just a number, not a rate. ${ }^{2}$

\footnotetext{
${ }^{2}$ A similar example: a clock is losing time at the rate of one second per month. Of course a clock which is losing time at the rate of one second per second is stopped.
} 
The population is getting older. Fewer older people are dying every year and every year fewer babies are being born. In fact, the average age of the population 5 years ago was 54. It is now 59. It has increased by one year per year. But by Olson's argument one year per year is just one, a number not a rate. Similarly, the average (modal) year of birth (or death) of the population has been changing from year to year. In 1640 it was 1615 . It is now, in 2013, 1960. In 2113 it will be 2030. (Likewise, the average year of death of the population in 1640 may have been 1670; in 1960, 2030; in 2110 maybe it will be 2220.) The rate of change will vary. Over a period of time it may be, say, two years per year. By Olson's reasoning this is two years divided by one year, which is the number two. But two is a number, not a rate.

These examples may be denied to be rates of change. However, they are certainly rates. But Olson's argument has it that they are not.

So the dynamic view of time cannot be shown to be incorrect by Olson's reasoning. The rate of change along a spatial dimension of a quantity which is measured in onedimensional spatial units is not a number. Likewise, the rate of change along the temporal dimension of a quantity which is measured in units of time is not a number.

It may be thought that this misses the point of Olson's objection to the dynamic view of time. In these cases, it may be said, although the units (centimetres, seconds etc.) do not cancel out this is because they represent variation in different dimensions. But when we say that time passes at the rate of one second per second we measure a second by that very same second. So the units do cancel out. Hence, Olson's objection should be understood as follows. In the proposition 'time passes at the rate of one second per second', 'one second per second' means 'one second divided by that very same second' - but this expression denotes the number one. But this cannot be Olson's objection since it is false that the expression 'one second divided by that very same second' denotes the number one, or anything else, since it 
is meaningless. In fact, division is a partially defined mathematical function which yields values for ordered pairs of numbers as argument. We can extend the definition, in conformity with Olson's thought, so that it is defined for objects that are not numbers so that, for example, Mars divided by Mars is one. And we can as easily extend it so that Mars divided by Venus (a different planet) is one, or that any second (e.g., the first after my birth) divided by itself or any other (e.g. the last of my life) is one. But without such an extension 'one second divided by that very same second' is no more meaningful than 'one cow divided by that very same cow', or 'one mile divided by that very same mile'. So it is not true that anything divided by itself, unless it is zero, is one.

There is no doubt that there is a fishiness about the proposition that time is passing at the rate of one second per second which is not shared by, for example, the proposition that the average age of the population is increasing by one year per year. But it does not help to identify the source of this fishiness to replace 'per' by 'divided by one'.

But, it may be said, in the unproblematic examples given variation in one dimension is measured against variation in another (when we draw the graphs we label the axes differently). So the units are not the same and so do not cancel out. But in the problematic cases they do.

However, this is wrong. The quantities are different in the unproblematic cases, for example, vertical height above sea level and horizontal distance from a point of origin, or average age (average temporal distance from birth) and year (distance from a selected temporal origin), or modal year of death (modal distance of time of death from a selected temporal origin) and year. But in each case the units are the same - centimetres in the first example, years in the second and third. Of course, these unproblematic cases contrast with the case of time's passage in that one cannot draw a graph to represent the latter with the axes 
differently labelled; each axis can only be labelled 'time from a selected temporal origin'. But this difference is not illuminated by appeal to the idea that 'per' means 'divided by one' and that numbers are not rates.

What is odd about asking 'How quickly is time passing?' is that it is analogous to asking 'How dense is space?'

This can be seen as follows. The question 'How quickly is time passing?' has as its only possible answer: 'one second per second'. But if the answer to the question is of the form ' $\mathrm{n}$ second(s) per second' asking the question must be equivalent to asking 'How quickly are the seconds passing?'Grammatically, then, asking how quickly time is passing is analogous to asking 'How quickly is that herd of cattle passing through the canyon?', the answer to which question will take the form 'X cows per second', e.g., '20 cows per second' or 'one cow per second'. ${ }^{3}$ So the question 'How quickly is time passing?', if it means anything, means the same as 'How quickly are the seconds passing?' And the statement 'time is passing' if it means anything, can only mean 'the seconds are passing', just as 'the herd is passing' can only mean 'the cows are passing, ${ }^{4}$ But 'how quickly are the seconds passing?' can only mean 'how many seconds are passing per second (per minute, per hour)?', i.e., how many seconds are there per second - to which the only possible answer is 'one second per second (60 per minute, 3,600 per hour)'. Again, the question how quickly time is passing is analogous to the question how quickly someone's heart is beating, i.e., how many beats are there in a second. This is in turn analogous to asking how dense some substance is. The answer to the former question, e.g., two beats per second, tells us how many heartbeats

\footnotetext{
${ }^{3}$ The question about the rate of passage of the herd may also be understood as asking for the rate, measured in metres per second, at which the cows are passing. Correspondingly, the question about the rate of time's passage may be understood as asking for the rate, measured in seconds per second, at which the seconds are passing. The only possible answer to this is: the seconds are (each second is) passing at the rate of one second per second. So we arrive at the same place as in the text, though via a different route.

${ }^{4}$ 'Time has passed so slowly since slowly since our last meeting', if it means anything, means the same as 'the years/days/minutes/seconds have passed so slowly since our last meeting'.
} 
occupy a time interval of one second. The answer to the second question, e.g., two grams per cubic centimetre, tells us what mass of the substance occupies a spatial volume of one cubic centimetre.

So to ask how quickly time is passing is analogous to asking 'How dense is space?' To ask this is to ask what quantity of space occupies a spatial region of one cubic centimetre, i.e., how many cubic centimetres of space there are per cubic centimetre of space. This question is formulated by replacing 'lead' by 'space' in the question 'What quantity of lead occupies a spatial region of one cubic centimetre?', but unlike the original question it is a meaningless one - to which it seems the only possible answer can be 'One cubic centimetre per cubic centimetre'. In fact the verbal substitution has destroyed meaning. ${ }^{5}$ In exactly the same way it seems that the only possible answer to the question how many seconds there are per second, which can be got from the question 'How many heartbeats are there per second?' by replacing 'heartbeats' by 'seconds', must be: one second per second. But in fact the verbal substitution has transformed a meaningful question into a meaningless one.

Another way of seeing that the question about time's passage is analogous to the meaningless question about the density of space is as follows. One can ask how many public houses there are per square mile in a city, or how many restaurants per mile along a highway. The answers may be fifty pubs per square mile and 30 restaurants per mile. These answers tell us how thick on the ground the pubs and restaurants are. The second may also be said to tell us the rate at which restaurants occur along the highway. If we ask how thick on the ground the ground is, however, the only possible answers are: one square mile per square mile and one mile per mile. The second answer may also be said to tell us the rate at which

\footnotetext{
${ }^{5}$ Perhaps the question about the density of space is not meaningless, but simply trivial in having only one possible answer. My point is that the question about the rate of time's passage has an exactly analogous status. I will continue to refer to this status as 'being meaningless'.
} 
miles occur along the highway - they pass (or one passes them) at the rate of one per mile. ${ }^{6}$ Asking how quickly time passes is analogous to asking how thick on the ground the ground is.

No appeal to the idea that that 'per' means 'divided by one ...' is needed to explain the oddity of the question 'How dense is space?' or 'How thick on the ground is the ground?' Nor then is it needed to explain the oddity of the question 'How quickly does time pass?' But the questions are precisely analogous, and precisely analogously meaningless. So the dynamic view of time, which requires the latter to be a sensible question, is mistaken. As the poet says, the truth is that we pass (at the (average) rate of so-and-so many million people per year) ${ }^{7}$; time stays.

\section{References}

Olson, E. 2009 The rate of time's passage. Analysis 69:3-9

Phillips, I. 2010 Rate abuse: a reply to Olson. Analysis 69:503-5

\footnotetext{
${ }^{6}$ I.e., quite slowly with a bored infant in the car.

${ }^{7}$ The average rate of death over, say, the last ten years will be so-and-so many people per year; more in times of war and famine, less in times of peace and abundance.
} 